\title{
Estándares para registrar señales de ecolocalización y construir bibliotecas de referencia de murciélagos en Colombia
}

\section{Standards for recording echolocation signals and building reference libraries of bats in Colombia}

\author{
Daniela Martínez-Medina ${ }^{(i)} \square$, Jefferson Sánchez ${ }^{(i)} \square$, Danny Zurc ${ }^{(i)} \square$, Francisco Sánchez ${ }^{(i)} \square$, \\ Aída Otálora- Ardila $\square$, Carlos Restrepo-Giraldo ${ }^{\square}$, Orlando Acevedo-Charry ${ }^{\square}$, \\ Fabián Hernández Leal ${ }^{(i)}$, Diego J. Lizcano $\square$
}

\section{Resumen}

La bioacústica permite monitorear murciélagos difícilmente detectados con métodos tradicionales, como las redes de niebla, y permiten obtener información sobre diferentes aspectos de la biología de las especies de estudio. En los últimos años se ha incrementado el interés por la aplicación de estos métodos y, en consecuencia, ha sido rápido el desarrollo de los equipos de grabación ultrasónica y las herramientas de análisis. Sin embargo, en el Neotrópico la bioacústica de murciélagos está en etapa de crecimiento en algunos países y apenas ahora está apareciendo en otros, por lo cual es necesario fortalecer este campo para estudiar la alta diversidad taxonómica y funcional de la región. En este contexto, es indispensable contar con bibliotecas de señales acústicas, que sirvan como referencia para identificar, validar y comparar las grabaciones de diferentes especies y localidades. Algunos países del Neotrópico han avanzado en este proceso y están construyendo estas bibliotecas de referencia. En Colombia, el trabajo a futuro implica un reto, debido a las más de 200 especies de murciélagos presentes. Este trabajo presenta las bases metodológicas para tomar grabaciones de referencia de murciélagos en Colombia y generar una guía para aquellos que se están iniciando en este campo de investigación.

Palabras clave. Bibliotecas de referencia. Chiroptera. Ecolocalización. Métodos bioacústicos. Métodos de grabación.

\begin{abstract}
Bioacoustic tools allow monitoring bats usually not detected with traditional methods, such as mist nets, and also provide information about different biological aspects of the species. The interest in applying these methods has increased in the last years, and consequently, there has been a rapid development of ultrasonic recording equipment and analysis tools. However, in the Neotropics, bat bioacoustics is emerging in some countries and just appearing in others; therefore it is necessary to reinforce this field to study the high taxonomic and functional diversity of the region. Therefore, it is essential to have bat call libraries that serve as a reference to validate and compare recordings from different species and localities. Some countries in the Neotropics have advanced in this process and are already building these reference libraries. In Colombia, the future work is challenging, due to the more than 200 bat species present. This paper establishes the methodological basis to obtain bat-reference calls in the Colombia and allow those who are just starting out in this research field.
\end{abstract}

Key words. Bioacoustic methods. Chiroptera. Echolocation. Recording methods. Reference libraries. 


\section{Introducción}

La mayoría de los murciélagos son capaces de ecolocalizar, es decir de emitir señales acústicas de alta frecuencia y utilizar sus ecos para formar una "imagen" en tres dimensiones del espacio (Altringham \& Fenton, 2003). En otras palabras, cuentan con un biosonar que les permite realizar complejas tareas como evitar obstáculos, identificar objetos estáticos como flores y frutos, o detectar presas potenciales en movimiento, como insectos en vuelo. Esta adaptación les ha permitido colonizar con éxito la noche y lograr una considerable diversificación que se refleja en las más de 1400 especies de murciélagos que se encuentran en el mundo (Wilson \& Mittermeier, 2019; Burgin et al., 2018).

Debido a las características temporales y espectrales de las señales que emiten los murciélagos en el ultrasonido, estas se pueden clasificar en tres fases: búsqueda de alimento, aproximación a una potencial fuente, y fase terminal (Griffin et al., 1960). Además de presentar "llamadas sociales" las cuales se han descrito como señales empleadas para interactuar con otros individuos (Knörnschild et al., 2012). Diferentes estudios han indicado que los sonidos producidos por los murciélagos presentan variabilidad ecológica (Barlow \& Jones, 1997; Barclay et al., 1999), geográfica (Jiang et al., 2015; López-Baucells et al., 2018), comportamental (Obrist, 1995), entre sexos (Puechmaille et al., 2014), edades (Jones \& Ransome, 1993; Siemers et al., 2005), y plasticidad frente a condiciones ambientales diferentes (Gillam \& McCracken, 2007).

Los murciélagos son el grupo de mamíferos más diverso Colombia (Ramírez-Chaves et al., 2016). Su estudio se ha realizado principalmente usando redes de niebla como método de captura. Sin embargo, la aparición y evolución de los detectores ultrasónicos, y sus precios progresivamente más asequibles, han permitido un incremento en el uso de herramientas bioacústicas para estudiar la diversidad en ambientes tropicales (Russo \& Voigt, 2016; Braun de Torrez et al., 2017; Brigham, 2018). Estas herramientas han adquirido mayor relevancia para la cuantificación y monitoreo de poblaciones y ensamblajes de aquellos murciélagos que generalmente no son detectados con métodos tradicionales (Meyer et al., 2011). Por lo tanto, los estudios acústicos son complementarios a los muestreos de murciélagos con redes de niebla, con trampas harpa o capturas con redes manuales (MacSwiney et al., 2008; Zamora-Gutierrez et al., 2016). Estudios acústicos de murciélagos han permitido obtener información sobre patrones de actividad (Bartonička \& Zukal, 2003; Kalcounis-Rüppell et al.,
2003; Meyer et al., 2004), identidad taxonómica (Barclay, 1983; Rodríguez \& Mora, 2006; Xu et al., 2008), uso de hábitat (Agosta, 2002; Rogers et al., 2006) y otros aspectos ecológicos de los murciélagos.

En años recientes los métodos de clasificación automatizada de señales de ecolocalización de los murciélagos han tenido un considerable desarrollo estadístico y computacional para la identificación de las especies (Stathopoulos et al., 2018; Heim et al., 2019). El uso de métodos estadísticos de clasificación y la asequibilidad de los dispositivos de detección ultrasónica proveen el potencial para implementar programas de monitoreo a escala regional, continental y global, así como para estudios estandarizados a largo plazo (Haase et al., 2018; Schmeller et al., 2018; Beason et al., 2019). La ventaja es que estos métodos de clasificación permiten analizar una gran cantidad de datos en un tiempo relativamente corto, aunque se ha cuestionado su precisión a la hora de diferenciar especies con señales de ecolocalización similares y el tipo de señales con las que se han entrenado los algoritmos de clasificación (Russo \& Voigt, 2016).

Para que los métodos de clasificación automática sean aplicables y funcionales en países biodiversos como Colombia, se requiere de un conocimiento básico y organizado de las señales acústicas de los murciélagos, que permitan calibrar correctamente los modelos y algoritmos de clasificación. Esto implica contar con una buena identificación taxonómica de cada una de las especies de murciélagos junto con las características de las señales acústicas que emiten. Por estas razones, es fundamental contar con bibliotecas de referencia de señales acústicas de una amplia serie de localidades y hábitats (López-Baucells et al.,2018), como soporte para la adecuada identificación, principalmente porque existen muchos otros factores que influyen en la variación de la estructura de las señales a nivel intraespecífico.

Si bien algunos países en Latinoamérica, como México (Zamora-Gutiérrez et al., 2020), Ecuador (Rivera-Parra \& Burneo, 2013) y Brasil (Arias-Aguilar et al., 2018; López-Baucells et al.,2016), han dado grandes pasos para construir estas colecciones, Colombia, aún no cuenta con una biblioteca de referencia para la identificación de murciélagos, a pesar de ser el segundo país en el mundo con mayor diversidad de estos organismos. En este trabajo se proponen estándares para el registro y almacenamiento de señales de ecolocalización de murciélagos colombianos. Esta propuesta tiene como objetivo establecer las bases metodológicas mínimas para la toma, procesamiento y almacenamiento de datos 
acústicos de murciélagos a nivel nacional. A continuación, se propone un marco de trabajo que plantea las bases para registrar, analizar y almacenar señales de ecolocalización de murciélagos en Colombia (Figura 1, Tabla 1).

Toma de datos en campo. Como el objetivo final de una biblioteca de referencia es permitir a futuro la identificación de las señales acústicas de murciélagos en vuelo libre bajo condiciones naturales (Zamora et al.,2020), es muy importante tener en cuenta los rasgos ecológicos y comportamentales de la especie que se quiere grabar. Con base en esto, se debe escoger la metodología más apropiada. A continuación, se presentan las metodologías de grabación más utilizadas con sus respectivas recomendaciones.

Cuarto de vuelo. Los encierros o cuartos de vuelo son espacios en los cuales se libera al murciélago para obtener grabaciones controladas. Pueden construirse con redes sintéticas sostenida por postes o tubos PVC, o pueden usarse construcciones abandonadas o invernaderos. Las grabaciones en encierro de vuelo tienen dos ventajas: 1) Se puede obtener el espécimen testigo para una colección científica. 2) Es posible tener varias grabaciones de un mismo individuo (Siemers, 2004).

Sin embargo, ya que las características de las señales son modificadas por los murciélagos en respuesta a las características específicas del espacio circundante, es posible que las grabaciones realizadas en encierros de vuelo estrechos o pequeños, contengan señales de ecolocalización similares a los tipos de señales que un murciélago emitiría dentro de un espacio cerrado o estructuralmente complejo ("cluttered") (Anexo 1). Esta es la mayor desventaja de esta técnica, puesto que es probable que en un encierro de vuelo pequeño los murciélagos no emitan el tipo de señal que usan en vuelo libre o en un espacio abierto (Parsons \& Szewczak, 2009; Anexo 1).

1. Toma de datos en campo

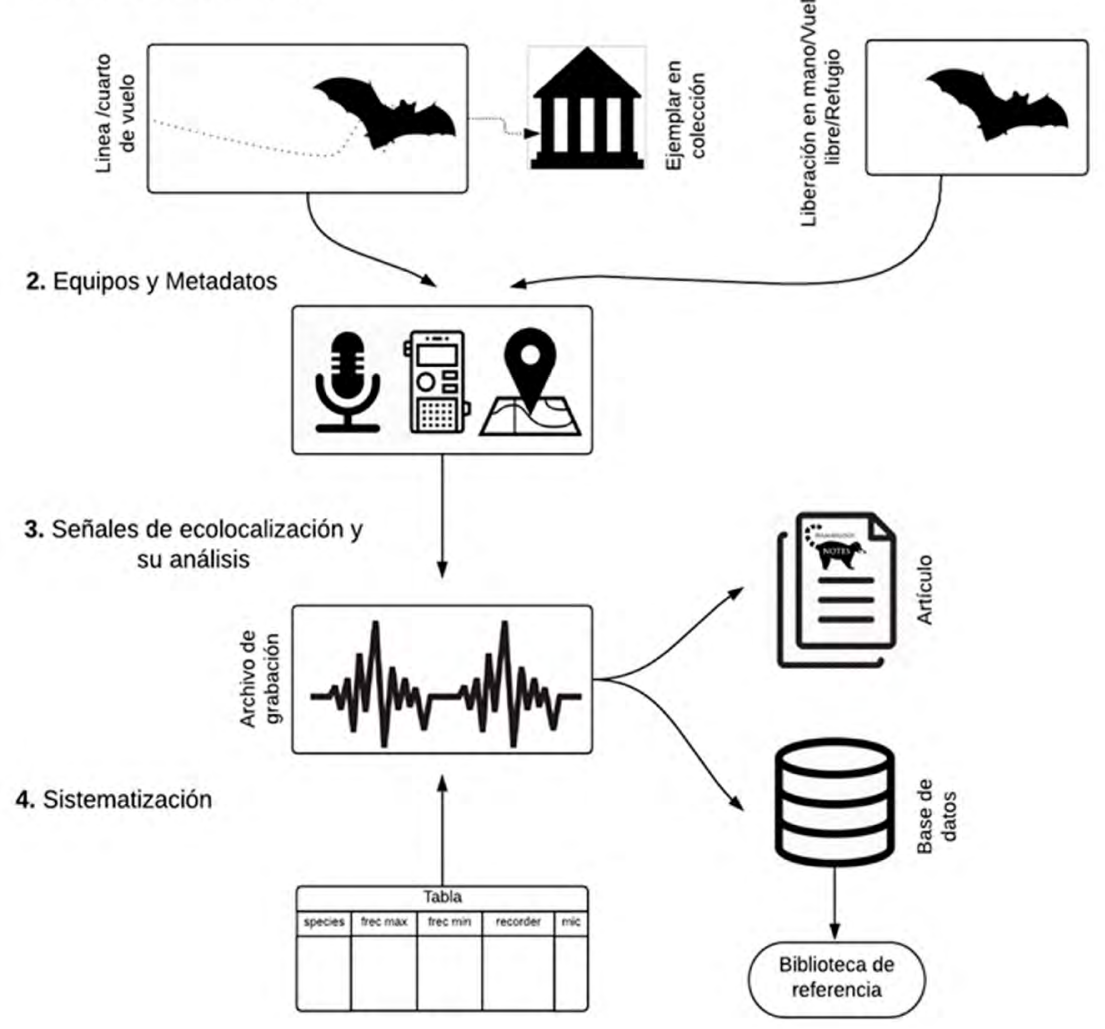

Figura 1. Marco de trabajo para registrar, analizar y almacenar señales de ecolocalización de murciélagos en Colombia.

Figure 1. Framework to record, analyze and store echolocation bat calls in Colombia. 
Otra desventaja surge de la superposición de la señal acústica emitida por el murciélago y el eco resultante de las superficies que componen el encierro, que puede afectar la calidad de la grabación. Es recomendable realizar una revisión del trabajo de Mora et al. (2004) para una mejor comprensión del fenómeno de superposición y enmascaramiento de las señales acústicas. Recomendaciones: Se deben grabar especies de murciélagos que vuelan en espacios estructuralmente complejos o especies con señales de intensidad baja; no es recomendable usar esta metodología en especies adaptadas a volar en espacios abiertos. Para construir el encierro se debe considerar un espacio lo suficientemente amplio que permita el desplazamiento del animal a lo largo del mismo y se deben usar materiales poco ecoicos. Las telas, cuanto más porosas y menos brillantes visualmente, reflejan menos las frecuencias. Por ejemplo, la tela de toldillo para insectos es menos tupida, por lo que produce menos reflexiones y el sonido es más limpio; sin embargo, la limitante sería el ruido exterior. Otro material adecuado sería el paño escorial, que aísla el ruido exterior, pero su manipulación en el campo es más complicada.

Cuerda de vuelo (Zip-line). Esta técnica consiste en extender una cuerda de nylon de pesca $(2 \mathrm{~mm}$ de calibre) entre dos puntos distanciados 10-30 m, y a una distancia mínima de $1 \mathrm{~m}$ del suelo. Sobre esta cuerda se introduce un aro metálico muy liviano a modo de guía, que estará atado a un hilo de nylon de menor calibre $(0.3 \mathrm{~mm})$, de 2 a $3 \mathrm{~m}$ de longitud, y en su extremo se dispone un collar de goma que se le colocará al individuo a referenciar (Szewczak, 2004). Esta técnica de grabación tiene varias ventajas: 1) Facilita que el murciélago alcance una velocidad y altura de vuelo estables en las que puede emitir señales de ecolocalización típicas, 2) permite recapturar el individuo y realizar múltiples grabaciones, hasta obtener una con buena calidad, y 3) se puede obtener el espécimen testigo para una colección científica. Por otro lado, sus desventajas están relacionadas con el cambio en la estructura de las señales de ecolocalización en individuos de la familia Molossidae y algunos de la familia Vespertilionidae (Parsons \& Szewczak, 2009). Recomendaciones: No todos los murciélagos alcanzan un vuelo estable al primer intento en la cuerda de vuelo, por lo que se necesita realizar varios intentos. De igual forma, algunos individuos rechazan el montaje o no vuelan, lo que impide obtener grabaciones. Por último, es importante realizar el montaje en un sitio abierto que le permita al murciélago volar y no enredarse con ningún tipo de vegetación.
Liberación manual (Hand release). Este método consiste en registrar las señales de ecolocalización de los murciélagos inmediatamente son liberados. Permite registrar señales representativas de especies de vuelo lento o murciélagos que se alimentan de presas estáticas (Parsons \& Szewczak, 2009). Una desventaja de este método constituye el hecho de que generalmente los murciélagos recién liberados no emiten señales que representen fielmente su comportamiento ecolocalizador típico cuando están en vuelo libre (Szewczak, 2004), aunque si se logra hacer adecuadamente, se pueden obtener señales más parecidas que las que se obtienen en cuarto de vuelo o en cuerda de vuelo. Las principales desventajas de esta metodología son: 1) no se obtiene un espécimen testigo para una colección científica y 2) solo se puede hacer una grabación. Otra dificultad asociada a este método es que se debe seguir el movimiento del murciélago una vez liberado, asegurando que la distancia entre éste y el detector sea la adecuada para que el micrófono tenga buen registro de las señales. Recomendación: Para compensar esta última dificultad, se puede incluir el uso de señaladores lumínicos (glow stick) para seguir el vuelo en la oscuridad y obtener secuencias de ecolocalización largas (Broders et al., 2004). Esta metodología es recomendada solo cuando el investigador tiene certeza sobre la identidad taxonómica de la especie.

Grabaciones cerca a refugios. Encontrar un refugio es una gran oportunidad para hacer grabaciones de referencia, puesto que se pueden obtener múltiples grabaciones de una especie en vuelo libre. De igual forma, el investigador cuenta con la posibilidad de capturar un espécimen para corroborar su identificación taxonómica e ingresar un espécimen a una colección científica. Una de las desventajas de esta metodología es que cuando la actividad de los murciélagos es muy alta, se puede registrar más de un individuo y las grabaciones pueden quedar saturadas, lo cual puede ser problemático al individualizar señales de un taxón para su análisis. Así mismo, se debe tener certeza de que las señales acústicas son grabadas cuando los murciélagos ya han entrado en la fase de búsqueda, para facilitar su identificación o comparación con otras grabaciones (Parsons et al., 2009).

Grabar murciélagos desde perchas. Estas grabaciones se hacen después de dejar a los individuos posados en un árbol, palmera o superficie donde el murciélago pueda percharse. La finalidad es registrar las señales emitidas una vez los murciélagos inicien el vuelo. La distancia del grabador puede variar y dependerá de la 
altura a la que el murciélago se ubique para volar. Este método es útil para especies que requieren de una altura considerable para lanzarse al vuelo como es el caso de las especies de la familia Molossidae, las cuales han sido de las más registradas con este método (Zamora et al., 2020). Recomendaciones: Identificar un árbol o palmera en un área abierta. El árbol debe tener pocas ramas y no presentar huecos o estructuras donde los murciélagos puedan ocultarse. El investigador debe estar atento con el sistema de detección encendido para hacer los registros al momento en que el murciélago tome el vuelo.

\section{Equipos y Metadatos.}

Equipos. La selección del equipo debe estar guiada por la pregunta que se quiere responder, el presupuesto con el que se cuenta, las especies que se quieren monitorear, y la capacidad de almacenamiento y análisis de datos, entre otros factores. Es recomendable hacer una revisión de la compilación de Brigham et al. (2002), donde se explican las diferencias de los equipos utilizados, sus capacidades y limitaciones. A continuación, se mencionan las características más relevantes de los diferentes equipos de detección y en el Anexo 2 se mencionan los fabricantes y los modelos más usados hasta el año 2020.

Los detectores de murciélagos heterodinos (Anexo 1), son los equipos menos costosos del mercado y proporcionan el método de identificación de especies más rápido, ya que su identificación se lleva a cabo en el campo y cuando hay una buena idea de las especies de la zona (Russ, 2012). Son equipos útiles para hacer un seguimiento en tiempo real de la actividad de los murciélagos, pero usualmente no incluyen un dispositivo de grabación, y cuando se le acopla uno, sus grabaciones suelen tener pobre resolución temporal y no retienen toda la información espectral de las señales de ecolocalización.

Existen sistemas de grabación basados en el registro de cruces por cero ("zero-crossing") (Anexo 1). Estos dispositivos sólo graban la frecuencia de mayor intensidad por unidad de tiempo, lo que implica que la representación gráfica de las señales de ecolocalización sea una serie de puntos, de los cuales se puede extraer poca información espectral y temporal, pero que ocupan poco espacio de almacenamiento.

Otros dispositivos de detección graban el espectro completo o "full spectrum" (Anexo 1), y cuentan con una alta resolución temporal y espectral. Este tipo de detectores usualmente cuentan con un dispositivo de grabación integrado y un sistema con disparador automático, que permite desplegar estaciones de monitoreo pasivo en campo por periodos prolongados. Adicionalmente, se ha argumentado que las grabaciones de señales acústicas realizadas con detectores de espectro completo son las más adecuadas para identificar especies (Corben, 2002).

Micrófono El potencial de detección de señales ultrasónicas depende de la sensibilidad del micrófono, la cual debe ser superior a los $10 \mathrm{kHz}$. Actualmente la mayoría de los dispositivos para detectar señales ultrasónicas cumplen con este requisito. Los micrófonos de estos dispositivos pueden ser omnidireccionales o unidireccionales, y su uso depende del interés de investigación, ya que los micrófonos omnidireccionales pueden ser usados para estudios de diversidad en los que se requiere registrar la mayor cantidad de especies posible, mientras que los unidireccionales se adaptan mejor a situaciones donde se requiere enfocar el área de detección del micrófono sobre un espacio reducido, como cuando se realizan experimentos en espacios cerrados.

Los micrófonos más comúnmente usados son: 1) micrófonos Piezo, que tienen un pico agudo de sensibilidad a frecuencias medias (aproximadamente $40 \mathrm{kHz}$ ); 2) micrófonos Electret, que son menos sensibles a frecuencias medias y más sensibles a 20 y $100 \mathrm{kHz}$, y 3) los micrófonos Capacitancia, que son más sensibles a frecuencias altas (> $80 \mathrm{kHz}$ ) (Russ, 2012). Los detectores que traen MEMS micrófonos tienen respuestas de frecuencia más consistentes entre los 40 y $60 \mathrm{kHz}$, con sensibilidad más baja a otras frecuencias, mientras que otros detectores tienen más de un micrófono, con el fin de incrementar la sensibilidad a través de un rango de frecuencias (Russ, 2012).

Tasa de muestreo Uno de los atributos fundamentales en la grabación de señales acústicas de alta frecuencia, es la tasa de muestreo (Anexo 1), la cual determina la frecuencia máxima obtenida para reconstruir una señal acústica. Por lo general, la tasa de muestreo es el doble de la mayor frecuencia contenida en el sonido de interés, de acuerdo con el teorema de Nyquist-Shannon (Nyquist, 1928; Shannon, 1949). Entonces, para murciélagos que emiten señales acústicas hasta de $125 \mathrm{kHz}$, la tasa de muestreo debe ser por lo menos de $250 \mathrm{kHz}$.

Formatos de grabación. Los dispositivos de grabación digital pueden grabar en diferentes formatos, pero el 
formato estándar es sin compresión Waveform (ej. wav); por lo anterior es recomendable usar este formato, ya que conserva toda la información del archivo de sonido; sin embargo, es el formato con el tamaño de archivo más grande de almacenamiento (Russ, 2012). Existen otros formatos en los que se comprimen los archivos de sonido y necesitan menos espacio de almacenamiento, pero estos pierden información (p. ej. mp3, WMA, AAC, ATRAC) (Russ, 2012), por lo que no es recomendable para identificar especies de murciélagos. El problema de almacenamiento se puede resolver a través de servidores online o realizando grabaciones de corta duración.

Metadatos. A cada archivo de audio grabado en campo se le deben asignar metadatos, i. e., información sobre el archivo de grabación (Tabla 1). La propuesta de metadatos incluida aquí se basa en los campos exigidos por el protocolo de la Colección de Sonidos Ambientales del Instituto Humboldt (IAvH-CSA), los cuales pueden ser luego transformados a formatos Darwin Core estandarizados (https://www.tdwg. org/standards/dwc/).
Señales de ecolocalización y su análisis. Las señales de ecolocalización emitidas por los murciélagos son moldeadas por diversas fuentes de variación, como la historia evolutiva, los mecanismos de procesamiento de la información y las necesidades sensoriales impuestas por el tipo de hábitat, la estrategia de búsqueda de alimento y la dieta (Schnitzler et al., 1994; Siemers et al., 2001). Adicionalmente, el comportamiento de ecolocalización puede dividirse generalmente en tres fases, indistintamente de la especie en cuestión: cuando un murciélago insectívoro aéreo busca alimento (fase de búsqueda), cuando localiza la presa y se aproxima a ella (fase de aproximación) y, finalmente, cuando el murciélago intenta capturar la presa, se da la emisión de la fase terminal o "feeding buzz" (Anexo 1; Schnitzler \& Kalko, 2001; Russ, 2012). Las señales emitidas en la fase de búsqueda son relativamente uniformes, presentando poca variabilidad en rasgos espectrales o temporales, mientras que en las fases de acercamiento o fase terminal ocurren cambios en dichos rasgos (Obrist, 1995; Schnitzler \& Kalko, 2001). Por esta razón, se recomienda hacer las mediciones correspondientes para identificar las especies en la fase de búsqueda (O’Farrell \& Miller, 1999).

Tabla 1. Campos de metadatos requeridos y recomendados en la grabación de señales acústicas emitidas por murciélagos de Colombia.

Table 1. Required and recommended metadata fields while recording echolocation bat signals in Colombia

\begin{tabular}{lll}
\multicolumn{1}{c}{ Campo } & Tipo & \multicolumn{1}{c}{ Descripción } \\
\hline Fecha y hora & Obligatorio & Fecha y hora de la grabación \\
\hline ID de la grabación & Obligatorio & Nombre dado al archivo, que lo diferencia de otras grabaciones \\
\hline Identidad del sitio & Obligatorio & $\begin{array}{l}\text { Nombre de la localidad específica o punto donde las grabaciones fueron reali- } \\
\text { zadas (vereda, finca) }\end{array}$ \\
\hline Coordenadas & Obligatorio & Latitud y Longitud \\
\hline Metodología & Obligatorio & $\begin{array}{l}\text { Bajo qué metodología se llevó a cabo la grabación (Cuarto de vuelo, cuerda de } \\
\text { vuelo o liberación manual) }\end{array}$ \\
\hline Equipo de grabación & Obligatorio & Referencia (marca y modelo) del detector utilizado, pasivo o activo etc. \\
\hline Marca y tipo de Micrófono & Obligatorio & Referencia, unidireccional, omnidireccional \\
\hline Ganancia & Obligatorio & Ninguna o número en dB \\
\hline Tasa de muestreo & Obligatorio & Número en kHz \\
\hline Formato archivo & Obligatorio & Ejemplos: wav, W4V \\
\hline Vecindad inmediata & Obligatorio & Descripción del sitio: Cerrado, abierto, borde, sobre agua \\
\hline Datos del individuo & Obligatorio & Especie y sexo \\
\hline Datos del individuo & Opcional & Estado de desarrollo \\
\hline Datos de Colección & Sugerido & Número de colección y nombre de la colección donde se depositó el individuo. \\
\hline
\end{tabular}


La identificación de especies con base en las señales de ecolocalización implica necesariamente que se procesen las grabaciones en el laboratorio, filtrando, segmentando y clasificando los archivos grabados (Caycedo-Rosales et al., 2013). Una vez clasificado y ordenado, el material se podrá visualizar en algún software comercial especializado para tal fin, como BatSound (Pettersson Elektronik AB, Uppsala, Suecia), Raven Pro (The Cornell Lab of Ornithology, Ithaca, NY, USA), Avisoft SAS Lab Pro (Avisoft Bioacoustics, Berlín, Alemania), BatExplorer (Elekon A.G, Suiza), BatScope (WSL, Suiza), Kaleidoscope (Wildlife Acoustics, Inc. Maynard, Massachusetts, USA), Anabat Insight (Titley Scientific, USA), entre otros. Otra opción para el análisis y visualización es usar softwares libres, como los paquetes SeeWave (Sueur et al., 2008), Bioacoustics (Marchal et al., 2020) o WarbleR (Araya-Salas \& Smith-Vidaurre, 2017), del lenguaje libre de programación R (R Core Team, 2018).
Las señales ultrasónicas o pulsos emitidos por los murciélagos pueden describirse a través de la medición de variables temporales, como la duración del pulso, y espectrales, como la frecuencia inicial, final o de máxima energía. Además, los pulsos pueden caracterizarse en función de su forma y estructura (Anexo 1). En cuanto a la forma, los pulsos pueden ser hiperbólicos, cóncavos, curvi-lineares, rectos, entre otros. Basados en la estructura, el pulso puede tener un solo elemento de frecuencia modulada (FM) o una combinación de segmentos de frecuencia modulada y frecuencia constante (FM-FC) (Figura 2). Se recomienda registrar un conjunto básico de parámetros acústicos para asegurar el éxito de la biblioteca de referencia: frecuencia inicial, final, máxima y mínima, de máxima energía, ancho de banda, la dirección de la modulación, duración de pulso, intervalo interpulso (Anexo 1, Figura 2).

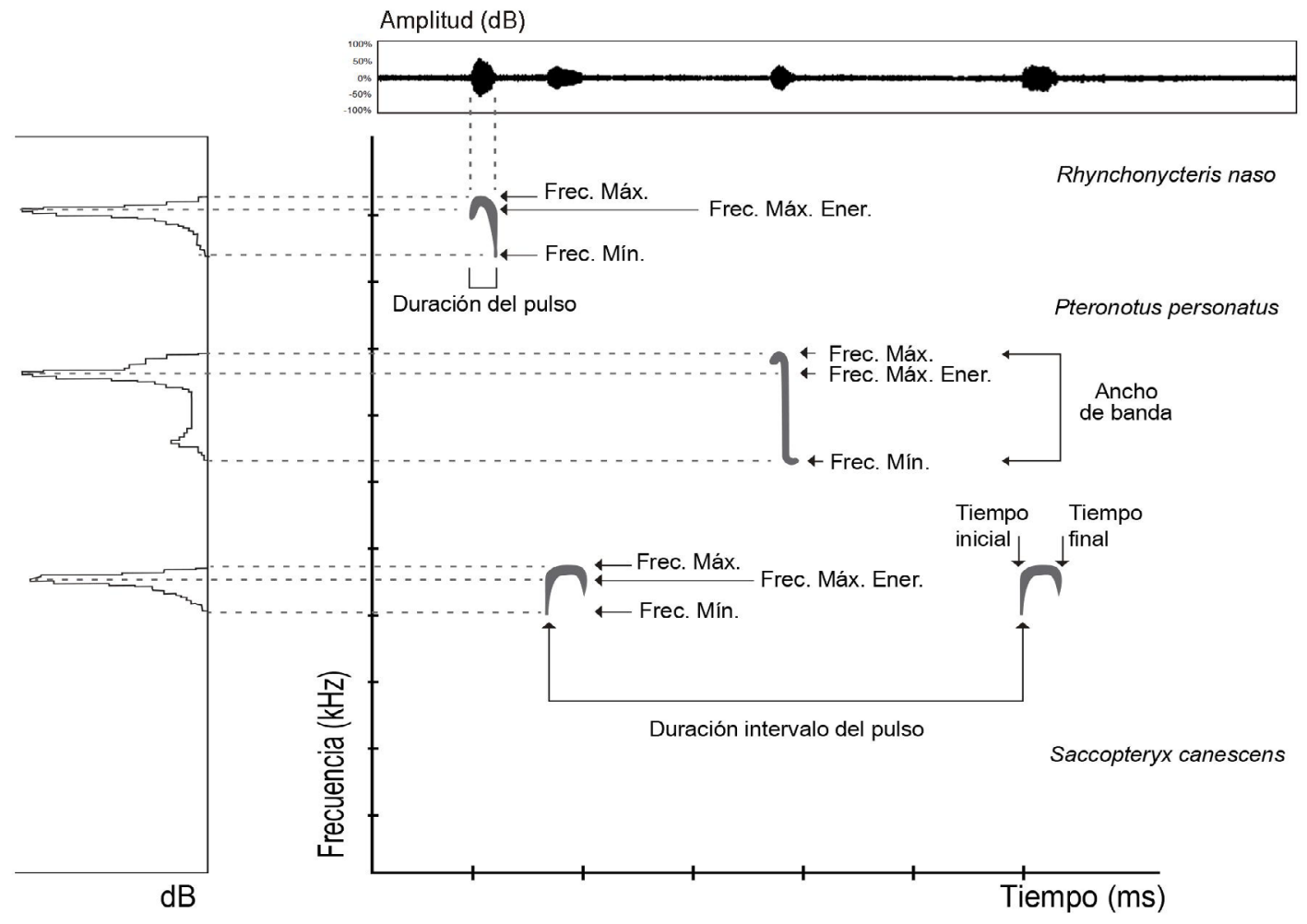

Figura 2. Algunos parámetros acústicos para caracterizar pulsos de ecolocalización. En la parte superior se muestra un oscilograma. A la izquierda se muestra un espectro de poder, a la derecha un espectrograma (Frec. Mín.= Frecuencia Mínima; Frec. Máx. Ener. = Frecuencia de Máxima Energía; Frec. Máx.= Frecuencia máxima)

Figure 2. Some acoustic parameters to characterize bat echolocation pulses. In the graph are shown power spectra (left), a spectrogram (right) and oscillogram (top). (Frec. Mín.= Minimum Frequency; Frec. Máx. Ener.= Peak Frequency; Frec. Máx. = Maximum Frequency) 
En el Anexo 1 se definen y señalan $\left(^{*}\right)$ los parámetros básicos que se deben extraer y medir de las señales de ecolocalización de los murciélagos, para generar una descripción confiable del comportamiento de ecolocalización de una especie.

Debe tenerse en cuenta que cuando se abre un archivo de audio es necesario asignar una "función de ventana", con lo cual se extrae el contorno de frecuencia vs. tiempo, con el propósito de reducir energía no deseada dentro de una banda útil de frecuencias (Fu \& Kloepper, 2018). Entre las funciones de ventana más utilizadas en el análisis de las señales de ecolocalización se encuentra la ventana Hamming (Jung et al., 2014; Anexo 1). Por esto se recomienda usar el mismo tipo de función de ventana en el programa usado para visualizar todos los espectrogramas (Avila-Flores \& Fenton, 2005) Anexo 1. Las medidas de los parámetros acústicos seleccionados deben realizarse sobre el armónico o los armónicos que presenten mayor energía. Es recomendable hacer las mediciones de las frecuencias desde el espectro de poder, y la duración del pulso y el intervalo de pulsos desde el oscilograma (Russo \& Jones, 2002). Como se mencionó anteriormente, una de las fuentes de variación que moldea el diseño de las señales de ecolocalización de los murciélagos es la historia evolutiva de cada especie y cada linaje, por lo que a continuación se presentan recomendaciones específicas para trabajar con algunas familias de murciélagos.

Emballonuridae. En esta familia los pulsos son multiarmónicos y generalmente la mayor energía se presenta en el segundo armónico (Barclay, 1983). Cada uno de los pulsos se caracteriza por ser de frecuencia constante (FC), de banda estrecha y con un barrido de frecuencia modulada (FM) corto al inicio y al final de cada pulso (Orozco-Lugo et al., 2013, Jung et al., 2007, Zurc et al. 2017). Algunas de las especies de esta familia presentan alternación de pulsos, es decir, exhiben un pulso bajo y un pulso alto (p. ej. Saccopteryx bilineata, S. leptura, y Diclidurus albus). Por lo tanto, es necesario medir los parámetros acústicos elegidos en ambos pulsos (Figura 3D).

Molossidae. La familia Molossidae tiene diseños de pulsos género-específicos y especie-específicos (Jung et al. 2014). El armónico de mayor energía es el primero (Mora et al., 2014). En las especies de esta familia cuyo comportamiento de ecolocalización se ha descrito hasta ahora, la alternancia en los pulsos siempre está presente (Anexo 1). Por lo tanto, es necesario medir las variables temporales y espectrales seleccionadas, tanto en el pulso bajo como el pulso alto. Para el caso de Molossus molossus y Nyctinomops laticaudatus, se ha registrado la alternancia de hasta tres pulsos (bajo, medio y alto); en este caso, lo recomendado es medir los tres pulsos (Jung et al., 2014; Figura 3A).

Vespertilionidae. Algunas especies de esta familia presentan la mayor energía en el primer armónico; otras presentan la misma energía en el primer y segundo armónico (Denzinger et al., 2001). Generalmente, los vespertiliónidos exhiben pulsos cortos de frecuencia modulada descendente. En la mayoría de las especies que presentan este comportamiento, el parámetro espectral más importante es la frecuencia mínima ( $\mathrm{O}^{\prime}$ Farrell \& Miller, 1999). Algunas especies de esta familia presentan variaciones en la emisión de señales de ecolocalización, dependiendo de la complejidad estructural del espacio por donde vuelan. Por ejemplo, especies de los géneros Myotis y Eptesicus exhiben cierta plasticidad en la emisión de sus pulsos de ecolocalización. En espacios cerrados, emiten pulsos FM descendentes de modulación rápida, y cuando pasan a ambientes más abiertos, los pulsos se modifican, reduciendo la velocidad de modulación y exhibiendo una estructura FM descendente, con un componente de frecuencia cuasiconstante (Barataud et al., 2013; Figura 3E).

Noctilionidae. Las especies de esta familia se caracterizan por tener una estructura de pulsos compuesta por segmentos de frecuencia constante (FC) finalizando con segmentos de frecuencia modulada (FM) descendente, los cuales se denominan "pulso alto", mientras que los pulsos que presentan un segmento breve de frecuencia cuasiconstante (FQC) al inicio, y que terminan en FM se les denomina "pulso bajo". La frecuencia de máxima energía se encuentra en el primer armónico. Las dos especies de este género pueden diferenciarse porque Noctilio albiventris tiene una mayor frecuencia de máxima energía (FME) en el componente cuasiconstante (67-76 $\mathrm{kHz}$ ) mientras que $N$. leporinus $(53-61 \mathrm{kHz})$ (Barataud et al., 2013; Arias-Aguilar et al., 2018; Figura 3C).

Phyllostomidae. La mayoría de las especies usan regularmente áreas con vegetación densa, como el sotobosque, y por lo general emiten señales de ecolocalización de alta frecuencia, banda ancha y con varios armónicos. La mayoría de los murciélagos de esta familia son llamados murciélagos "susurradores", debido a la baja intensidad con la que emiten sus señales de ecolocalización. Esto dificulta su registro, a menos 
que emitan a una corta distancia del detector (Fenton et al., 2001). Las principales limitantes para implementar estudios acústicos sobre la familia Phyllostomidae son la baja especificidad de los diseños de señal, la baja amplitud (Anexo 1) y la alta frecuencia de estas señales (Yoh et al., 2020). En esta familia hay pocos casos de especialización en el diseño de las señales acústicas, y puede que esto se deba probablemente a que muchas especies usan el olfato o la visión en combinación con la ecolocalización para ubicar a sus objetivos (Leiser-Miller et al., 2020).

Mormoopidae. Esta familia se caracteriza por emitir usualmente pulsos con componentes multiarmónicos, siendo el segundo armónico aquel sobre el cual se concentra la mayor cantidad de la energía de la señal (Arias-Aguilar et al., 2018). Las especies presentan patrones en la estructura de los pulsos que son relativamente fáciles de distinguir. Pteronotus davyi y $P$. gymnonotus tienen una forma de pulso similar, que sigue una forma de " $\mathrm{z}$ " alargada, como la describen O'Farrell \& Miller (1999). Este patrón se caracteriza por presentar una FC inicial muy corta, seguida de una FM descendente, con alrededor de $15 \mathrm{kHz}$ de ancho de banda, y finaliza con una FC corta. Pteronotus parnellii emite señales con un ciclo de trabajo alto (Fenton et al., 2012) y sus pulsos son largos, de frecuencia constante, con segmentos de FM muy cortos al inicio (ascendente) y al final de la señal (descendente) (Barataud et al., 2013). Finalmente, las señales de ecolocalización de Mormoops megalophylla se caracterizan por carecer del componente FC, presentando sólo el componente de estructura FM descendente (O'Farrell \& Miller,1999).

Thyropteridae. Los pulsos emitidos por las especies de esta familia tienen una estructura FM descendente y son de corta duración (Barataud et al., 2013; Arias-Aguilar et al., 2018). Algunas especies pueden presentar la FME en el primer armónico y otras en el segundo (Barataud et al., 2013). Los sonidos emitidos por estas especies son de baja intensidad y se han registrado a distancias relativamente cortas del micrófono (1-3 m; Fenton et al., 1999; Tschapka et al., 2000). Los datos sobre las características del comportamiento de ecolocalización para esta familia son escasos.

Natalidae. Esta familia se caracteriza por emitir señales de ecolocalización con estructura FM descendente, con una alta inclinación o PLM, (Periodo Linealmente Modulado) y con un segmento terminal FQC corto. Así mismo, la duración del pulso es corta (<4 ms;
Arias-Aguilar et al., 2018; Barataud et al., 2013; Rydell et al., 2002; Sánchez et al., 2017). La frecuencia de máxima energía se encuentra en el segundo armónico, arriba de 100 kHz (Arias-Aguilar et al., 2018; Rydell et al., 2002). Al igual que las especies de la familia Thyropteridae, las grabaciones de las señales de ecolocalización de los murciélagos de esta familia, solo son bien registrados si se graban muy cerca al micrófono, ya que, por su baja intensidad, las señales tienden a atenuarse rápido, impidiendo que los parámetros acústicos más relevantes sean capturados por el dispositivo de detección (Rydell et al., 2002).

Furipteridae. Los pulsos de ecolocalización de las especies de esta familia consisten en barridos cortos de banda ancha modulada, de corta duración (2.3-4.7 ms), con una frecuencia mínima entre 67 y $135 \mathrm{kHz}$, una frecuencia máxima entre 150 y $191 \mathrm{kHz}$ y un pico de frecuencia entre 75 y 161 kHz (Falcão et al., 2015), no obstante, los pulsos pueden llegar hasta los $210 \mathrm{kHz}$, ante la presencia de artefactos muestreales en la grabación.

Finalmente, para describir los parámetros acústicos de una especie, es recomendable contar con grabaciones de varios individuos, para poder caracterizar la variación intraespecífica de dichos parámetros, y así tener una muestra representativa de la población. Es necesario medir una cantidad considerable de pulsos en fase de búsqueda por secuencia analizada (más de 20), ojalá de diferentes secuencias, seleccionando aquellos pulsos donde los parámetros acústicos permanecen poco variables. Se debe indicar el número de individuos, secuencias y pulsos usados para extraer las medidas.

Sistematización. Es necesario que todos los registros acústicos obtenidos estén organizados de forma que se permita su gestión y administración, lo que permitirá articular a diferentes investigadores e iniciativas de monitoreo. Para esto, sugerimos seguir un formato unificado de base de datos que pueda "enlazarse" con diferentes motores de bases de datos (ej. Postgres, Mongo, MySQL), como lo permite el formato Darwin Core (Darwin Core Task Group, 2009). El formato Darwin Core está compuesto por información estandarizada de taxonomía, lugares y eventos de registros de biodiversidad, y es la matriz de datos unificada en repositorios nacionales (p. ej. SiB Colombia) e internacionales (GBIF), por lo que constituye un excelente estándar a seguir por los investigadores de bioacústica de murciélagos colombianos. 


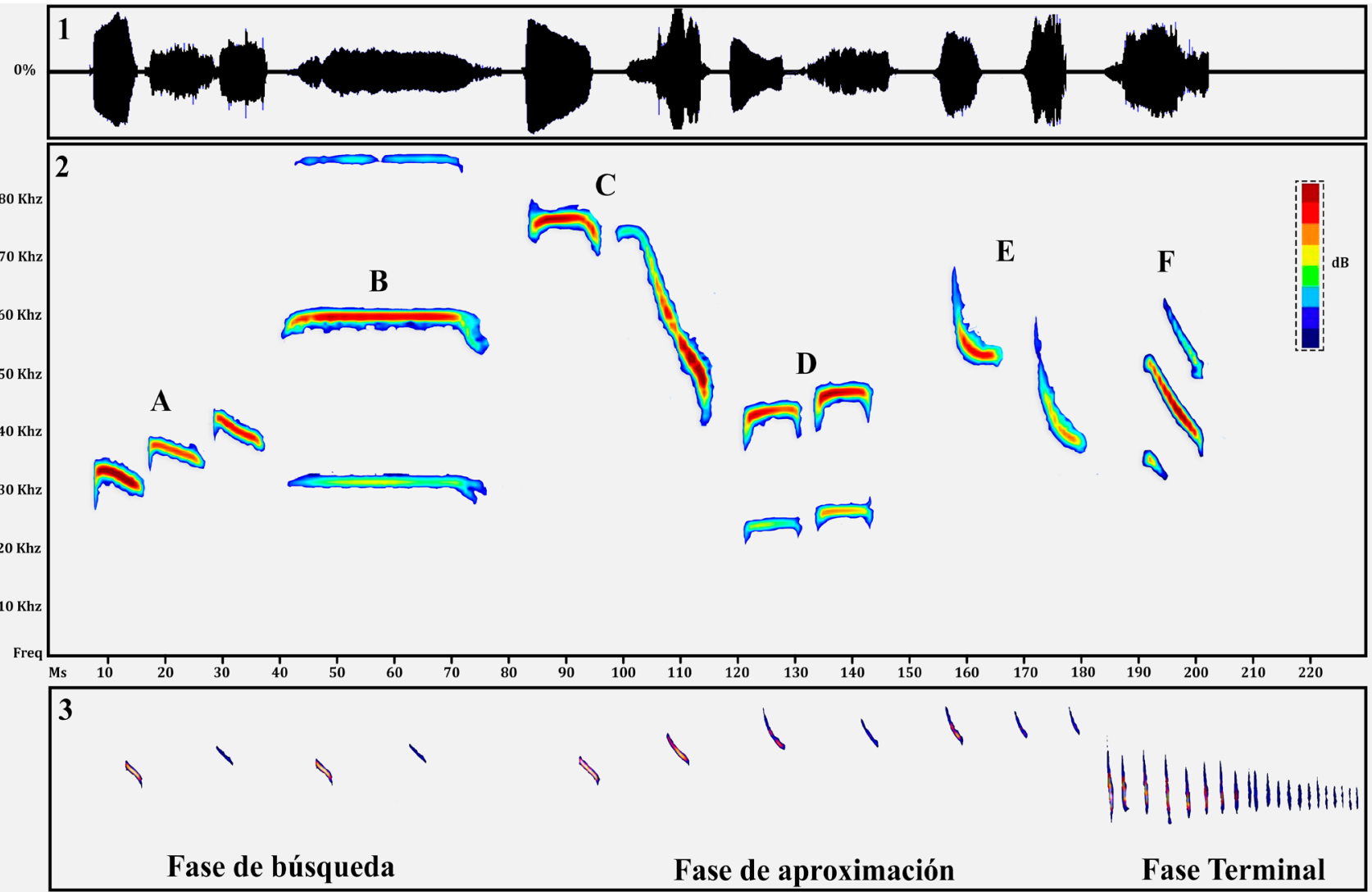

Figura 3. Ejemplos de parámetros acústicos para caracterizar pulsos de ecolocalización. 1, oscilograma; 2, espectrograma: pulsos característicos de algunas familias de murciélagos: A, Molossidae; B, Mormoopidae C, Noctilionidae; D, Emballonuridae; E, Vespertilionidae F, Phyllostomidae; 3, fases de ecolocalización de Molossus molossus.

Figure 3. Examples of acoustic parameters to characterize bat echolocation pulses. 1, oscillogram; 2, spectrogram: typical calls emitted by some bat families: A, Molossidae; B, Mormoopidae; C, Noctilionidae; D, Emballonuridae; E, Vespertilionidae; F, Phyllostomidae; 3, echolocation phases in Molossus molossus.

Recomendación: Al igual que sucede con los especímenes que se recolectan en campo, las grabaciones ultrasónicas con señales de ecolocalización de murciélagos también deben ser procesadas para su correcta sistematización. Este procesamiento implica cortar los archivos de audio que contiene la información relevante para analizar: dada la probabilidad de detección de cada especie, un archivo de audio debe contener al menos tres señales de ecolocalización para calcular parámetros temporales, como intervalos interpulso. Cuando la probabilidad de detección lo permite, en un archivo de audio quedarán registradas las distintas fases del comportamiento ecolocalizador (búsqueda, aproximación y terminal); de este modo, la duración máxima del archivo de audio será equivalente a la de las tres fases, incluyendo un par de pulsos posteriores al final de la fase terminal, cuando sea posible.
Adicionalmente, solo deben considerarse archivos de audio con señales de ecolocalización que posean una relación señal-ruido lo suficientemente buena para poder distinguir las señales de interés del ruido de fondo, lo que facilita la medición de los parámetros acústicos relevantes. Para esto se debe visualizar el espectro de poder del audio y verificar que haya una diferencia de al menos $25 \mathrm{~dB}$ entre el espectro de poder máximo del ruido de fondo y el espectro de poder mínimo de las señales de interés. Si la diferencia entre estos espectros de poder no sobrepasa los $25 \mathrm{~dB}$ no es recomendable usar ese archivo de audio, pues la medición de parámetros como la intensidad o las frecuencias pico puede verse afectada, y los valores medidos podrían no ser confiables. 


\section{Conclusiones}

Establecer estándares para registrar las señales de ecolocalización de murciélagos es el primer paso para construir bibliotecas de referencia en cada país. Esto evita duplicar esfuerzos, y permite que todas las grabaciones sean útiles, facilitando la extracción de los parámetros acústicos de interés que integrarán la biblioteca de referencia.

Los dispositivos disponibles actualmente para la detección y grabación de las señales de ecolocalización de murciélagos tienen ventajas y desventajas. Por ello no se recomienda el uso específico de un tipo o marca de detector ultrasónico, micrófono o grabadora. Sin importar cuál sea el equipo, es crucial que todos los investigadores que tengan grabaciones ultrasónicas de murciélagos puedan compartirlas junto con el conjunto de metadatos asociados a éstas. Se recomienda que las grabaciones se hagan en espectro completo (full spectrum) y que se registren todos los detalles técnicos y de configuración del detector o la grabadora, tales como ganancia en $\mathrm{dB}$, tasa de muestreo, tipo de archivo de almacenamiento, tiempo de grabación, duración de archivos, tiempo del disparador automático, entre otros. En los casos en los que el detector ultrasónico no este equipado para realizar grabaciones, es relevante registrar el modelo y marca de la grabadora y el micrófono o detector.

El método de monitoreo acústico en campo, el registro de los metadatos de campo, y el registro de referencia de las señales de ecolocalización dependen de la especie a tratar. Por ello, es recomendable conocer previamente los detalles del comportamiento de vuelo y forrajeo de cada especie, con el fin de registrar de manera adecuada el comportamiento de ecolocalización con todas sus variaciones.

Los parámetros acústicos que se miden varían para cada especie. Sin embargo, es importante registrar parámetros básicos cuando se describe el comportamiento ecolocalizador de una especie nueva. En lo posible, se deben capturar individuos de distintas especies y grabarlos de manera controlada, haciendo uso de al menos una de las técnicas de grabación mencionadas. Esto permitirá identificar y describir las señales acústicas propias de las especies presentes en el lugar de estudio.

Es importante que los investigadores envíen sus grabaciones junto con los metadatos a la Colección de Sonidos Ambientales del Instituto Humboldt; si bien es cierto que existen diferentes iniciativas en el país que están generando esta información en Colombia, actualmente esta es la única colección accesible en el país.

\section{Referencias}

Agosta, S. J. (2002). Habitat use, diet and roost selection by the big brown bat (Eptesicus fuscus) in North America: a case for conserving an abundant species. Mammal Review, 32(3), 179-198.

https:/ / doi.org/10.1046/j.1365-2907.2002.00103.x

Altringham, J. D. \& Fenton, M. B. (2003). Sensory ecology and communication in the Chiroptera. En Kunz, T.H \& Fenton, M.B. (Eds.). Bat Ecology. Pp. 90-127. Chicago: The University of Chicago Press.

Araya-Salas, M., Smith-Vidaurre, G. (2017). “warbleR: an $r$ package to streamline analysis of animal acoustic signals."

http:/ / doi.org/10.1111/2041-210X.12624

Arias-Aguilar, A., Hintze, F., Aguiar, L.M.S., Rufray, V., Bernard, E. \& Ramos, P. (2018). Who's calling? Acoustic identification of Brazilian bats. Mammal Research, 63(3), 231-253.

https:// doi.org/10.1007/s13364-018-0367-z

Avila-Flores, R. \& Fenton, M. B. (2005). Use of spatial features by foraging insectivorous bats in a large urban landscape. Journal of Mammalogy, 86(6), 11931204.

https:// doi.org/10.1644/04-MAMM-A-085R1.1

Barataud, M., Giosa, S., Leblanc, F., Rufray, V., Disca, T., Tillon, L., Delaval, M., Haquart, M. \& Dewynter, M. (2013). Identification et écologie acoustique des chiroptères de Guyane française. Le Rhinolphe, 19 (1), 103-145.

Barclay, R. M. (1983). Echolocation calls of emballonurid bats from Panama. Journal of Comparative Physio$\log y, 151(4), 515-520$.

https:/ / doi.org/10.1007/BF00605468

Barclay, R. M., Fullard, J. H., \& Jacobs, D. S. (1999). Variation in the echolocation calls of the hoary bat (Lasiurus cinereus): influence of body size, habitat structure, and geographic location. Canadian Journal of Zoology, 77(4), 530-534.

Barlow, K. E., \& Jones, G. (1997). Differences in songflight calls and social calls between two phonic types of the vespertilionid bat Pipistrellus pipistrellus. Journal of Zoology, 241(2), 315-324.

https:// doi.org/10.1111/j.1469-7998.1997.tb01962.x

Bartoničcka, T., \& Zukal, J. (2003). Flight activity and habitat use of four bat species in a small town revealed by bat detectors. Folia Zoologica, 52(2), 155-166. 
Beason, R. D., Riesch, R., \& Koricheva, J. (2019). AURITA: an affordable, autonomous recording device for acoustic monitoring of audible and ultrasonic frequencies. Bioacoustics, 28 (4), 381-396.

https:/ / doi.org/10.1080/09524622.2018.1463293

Braun de Torrez, E. C., Wallrichs, M. A., Ober, H. K., \& McCleery, R. A. (2017). Mobile acoustic transects miss rare bat species: implications of survey method and spatio-temporal sampling for monitoring bats. PeerJ, 5, e3940.

https://doi.org/10.7717/peerj.3940

Brigham, R. M., Kalko, E. K. V., Jones, G., Parsons, S., \& Limpens, H. J. G. A. (2002). Bat Echolocation Research: Tools, Techniques and Analysis. Austin, Texas: Conservation International. $174 \mathrm{pp}$.

Brigham, R. M. (2018). Learning to listen: a primer on bat echolocation research. Canadian Journal of Zoo$\log y, 96(9)$, 3-4.

https:/ / doi.org/10.1139/cjz-2018-0060

Broders, H. G.,Findlay,C. S., \& Zheng, L.(2004). Effects of clutter on echolocation call structure of Myotis septentrionalis and M. lucifugus. Journal of Mammalogy, 85(2), 273-281.

Burgin, C.J., Colella, J.P., Kahn, P.L., \& Upham, N.S. (2018). How many species of mammals are there? Journal of Mammalogy, 99(1), 1-11.

Caycedo-Rosales, P.C., Ruiz-Muñoz, J.F. \& Orozco-Alzate, M. (2013). Reconocimiento automatizado de señales bioacústicas: una revisión de métodos y aplicaciones. Ingeniería y Ciencia, 9(18), 171-195.

Corben, C. (2002). Zero-crossings analysis for bat identification: An overview. En Brigham, R. M., Kalko, E. K. V., Jones, G., Parsons, S., \& Limpens, H. J. G. A. (Eds.). Bat Echolocation Research: Tools, Techniques and Analysis. Pp: 95-107. Austin, Texas: Conservation International.

Corben, C. (2003). How Anabat works. The Australasian Bat Society Newsletter, 20, 19-23.

Darwin Core Task Group. (2009). Darwin Core text guide. Biodiversity Information Standards (TDWG).

http:/ / rs.tdwg.org/dwc/terms/guides/text/

Denzinger, A., Siemers, B. M., Schaub, A., \& Schnitzler, H. U. (2001). Echolocation by the barbastelle bat, Barbastella barbastellus. Journal of Comparative Physiology-A Sensory, Neural, and Behavioral Physiology, 187(7), 521-528.

https:/ / doi.org/10.1007/s003590100223

Falcão, F., Ugarte-Núñez, J. A., Faria, D., \& Caselli, C. B. (2015). Unravelling the calls of discrete hunters: acoustic structure of echolocation calls of furipterid bats (Chiroptera, Furipteridae). Bioacoustics, 24(2), 175-183. https:/ / doi.org/10.1080/09524622.2015.1017840
Fenton, M.B., Rydell, J., Vonhof, M.J., Eklöf, J., \& Lancaster, W.C. (1999). Constant-frequency and frequency-modulated components in the echolocation calls of three species of small bats (Emballonuridae, Thyropteridae, and Vespertilionidae). Canadian Journal of Zoology, 77(12), 1891-1900.

https:// doi.org/10.1139/ z99-168

Fenton, M. B., Bouchard, S., Vonhof, M. J., \& Zigouris, J. (2001). Time-expansion and zero-crossing period meter systems present significantly different views of echolocation calls of bats. Journal of Mammalogy, 82(3), 721-727.

https:// doi.org/10.1644/1545-1542

Fenton, M.B., Faure, P.A. \& Ratcliffe, J.M. (2012). Evolution of high duty cycle echolocation in bats. Journal of Experimental Biology, 215(17), 2935-2944.

https:/ / doi.org/10.1242/jeb.073171

Fu, Y., \& Kloepper, L. N. (2018). A systematic method for isolating, tracking and discriminating time-frequency components of bat echolocation calls. The Journal of the Acoustical Society of America, 143(2), 716-726.

https:/ / doi.org/10.1121/1.5023205

Gillam, E. H., \& McCracken, G. F. (2007). Variability in the echolocation of Tadarida brasiliensis: effects of geography and local acoustic environment. Animal Behaviour, 74(2), 277-286. https:/ / doi.org/10.1016/j. anbehav.2006.12.006

Griffin, D. R., Webster, F. A., \& Michael, C. R. (1960). The echolocation of flying insects by bats. Animal behaviour, 8(3-4), 141-154.

Haase, P., Tonkin, J. D., Stoll, S., Burkhard, B., Frenzel, M., Geijzendorffer, I. R., Häuser, C., Klotz, S., Kühn, I., McDowell, W.H., Mirti, M., Müller, F., Musche, M., Penner, J., Zacharias, S., \& Schmeller, D. S. (2018). The next generation of site-based longterm ecological monitoring: Linking essential biodiversity variables and ecosystem integrity. Science of the Total Environment, 613, 1376-1384.

https:// doi.org/10.1016/j.scitotenv.2017.08.111

Heim, O., Heim, D. M., Marggraf, L., Voigt, C. C., Zhang, X., Luo, Y., \& Zheng, J. (2019). Variant maps for bat echolocation call identification algorithms. Bioacoustics, 1-15.

https:/ / doi.org/10.1080/09524622.2019.1621776

Jiang, T., Wu, H., \& Feng, J. (2015). Patterns and causes of geographic variation in bat echolocation pulses. Integrative Zoology, 10(3), 241-256.

https:/ / doi.org/10.1111/1749-4877.12129

Jones, G., \& Ransome, R. D. (1993). Echolocation calls of bats are influenced by maternal effects and chan- 
ge over a lifetime. Proceedings of the Royal Society B: Biological Sciences, 252(1334), 125-128.

https:/ / doi.org/10.1098/rspb.1993.0055

Jung, K., Kalko, E. A. V., \& Von Helversen, O. (2007). Echolocation calls in Central American emballonurid bats: signal design and call frequency alternation. Journal of Zoology, 272(2), 125-137.

https:/ / doi.org/10.1111/j.1469-7998.2006.00250.x

Jung K, Molinari J., \& Kalko, E.K.V. (2014). Driving factors for the evolution of species-specific echolocation call design in new world free-tailed bats (Molossidae). PLoS One, 9(1).

https:/ / doi.org/10.1371/journal.pone.0085279

Kalcounis-Rüppell, M. C., Brown, T. J., Handford, P. T., \& Ojeda, R. A. (2003). Preliminary notes on bat activity and echolocation in northwestern Argentina. Mastozoología Neotropical, 10(2), 331-339.

Knörnschild, M., Jung, K., Nagy, M., Metz, M., \& Kalko, E. (2012). Bat echolocation calls facilitate social communication. Proceedings of the Royal Society B. Biological Sciences, 279(1748), 4827-4835.

https:/ / doi.org/10.1098/rspb.2012.1995

Leiser-Miller, L. B., Kaliszewska, Z. A., Lauterbur, M. E., Mann, B., Riffel, J. A., \& Santana, S. E. (2020). A fruitful endeavor: Scent cue and echolocation behavior used by Carollia castanea to find fruit. Integrative Organismal Biology, 2(1), obaa007.

https:/ / doi.org/10.1101/532614

López-Baucells, A., Torrent, L., Rocha, R., Pavan, A. C., Bobrowiec, P. E. D., \& Meyer, C. F. (2018). Geographical variation in the high-duty cycle echolocation of the cryptic common mustached bat Pteronotus cf. rubiginosus (Mormoopidae). Bioacoustics, 27(4), 341-357.

https:/ / doi.org/10.1080/09524622.2017.1357145

MacSwiney, M. C., Clarke, F. M., \& Racey, P. A. (2008). What you see is not what you get: the role of ultrasonic detectors in increasing inventory completeness in Neotropical bat assemblages. Journal of Applied Ecology, 45(5), 1364-1371.

https:/ / doi.org/10.1111/j.1365-2664.2008.01531.x

Marchal J., F. Fabianek, C. Scott, C. Corben, D. Riggs, P. Wilson, Wildlife Acoustics, inc. (2020). Bioacoustics package.

https:/ / cran.r-project.org/web/packages/bioacoustics/bioacoustics.pdf

Meyer, C. F., Schwarz, C. J., \& Fahr, J. (2004). Activity patterns and habitat preferences of insectivorous bats in a West African forest-savanna mosaic. Journal of Tropical Ecology, 20(4), 397-407.

https:/ / doi.org/10.1017/S0266467404001373
Meyer, C. F. J., Aguiar, L. M. S., Aguirre, L. F., Baumgarten, J., Clarke, F. M., Cosson, J.-F., \& Kalko, E. K. V. (2011). Accounting for detectability improves estimates of species richness in tropical bat surveys. Journal of Applied Ecology, 48(3), 777-787.

https:// doi.org/10.1111/j.1365-

Mora, E. C., Macías, S., Vater, M., Coro, F., \& Kössl, M. (2004). Specializations for aerial hawking in the echolocation system of Molossus molossus (Molossidae, Chiroptera). Journal of Comparative Physiology A, 190(7), 561-574.

Nyquist, H. (1928). Certain topics in telegraph transmission theory. Trans AIEE, 47:617-644.

Obrist, M. K. (1995). Flexible bat echolocation: the influence of individual, habitat and conspecifics on sonar signal design. Behavioral Ecology Sociobiology, 36(3), 207-219.

https:// doi.org/10.1007/BF00177798

O'Farrell M. J., \& Miller, B.W. (1999). Use of vocal signatures for the inventory of free-flying neotropical bats. Biotropica, 31(3), 507-516.

https:// doi.org/10.1111/j.1744-7429.1999.tb00394.x

Orozco-Lugo, L., Guillén-Servent, A., Valenzuela-Galván, D., \& Arita, H. T. (2013). Descripción de los pulsos de ecolocalización de once especies de murciélagos insectívoros aéreos de una selva baja caducifolia en Morelos, México. Therya, 4(1), 33-46.

Parsons, S., \& Szewczak, J.M. (2009). Detecting, recording, and analyzing the vocalizations of bats. En Kunz, T.H \& Parsons, S. (Eds.). Ecological and Behavioral Methods for the Study of Bats. Pp. 91-111. Baltimore, Maryland Second. Johns Hopkins University Press.

Puechmaille, S. J., Borissov, I. M., Zsebok, S., Allegrini, B., Hizem, M., Kuenzel, S., ... \& Siemers, B.M. (2014). Female mate choice can drive the evolution of high frecuency echolocation in bats: a case study with Rhinolophus mehelyi. PLos One, 9(7), e103452.

R Core Team. (2018). R: A language and environment for statistical computing. Vienna, Austria: R Foundation for Statistical Computing. Recuperado de http:/ / www.r-project.org/.

Ramírez-Chaves, H., Suárez-Castro, A., \& González-Maya, J. F. (2016). Cambios recientes a la lista de los mamíferos de Colombia. Notas Mastozoológicas, 3(1), 1-20.

https:/ / doi.org/2382-3704

Rivera-Parra, P., \& Burneo, S. F. (2013). Primera biblioteca de llamadas de ecolocalización de murciélagos del Ecuador. Therya, 4(1), 79-88.

http:/ / doi.org/10.12933/therya-13-104 
Rodríguez, A., \& Mora, E. C. (2006). The echolocation repertoire of Eptesicus fuscus (Chiroptera: Vespertilionidae) in Cuba. Caribbean Journal of Science, 42(1), 121.

Rogers, D. S., Belk, M. C., González, M. W., \& Coleman, B. L. (2006). Patterns of habitat use by bats along a riparian corridor in northern Utah. The Southwestern Naturalist, 51(1), 52-58.

Rojas, V. G., Loeb, S. C., \& O'Keefe, J. M. (2019). False-positive occupancy models produce less-biased occupancy estimates for a rare and elusive bat species. Journal of Mammalogy. 100(1), 212-222.

https:/ / doi.org/10.1093/jmammal/gyy162

Russ, J. (2012). British bat calls: A guide to species identification. Pelagic Publishing. 183 pp.

Russo, D., \& Jones, G. (2002). Identification of twenty-two bat species (Mammalia: Chiroptera) from Italy by analysis of time-expanded recordings of echolocation calls. Journal of Zoology, 258(1), 91-103. https:/ / doi.org/10.1017/S0952836902001231

Russo, D., \& Voigt, C. C. (2016). The use of automated identification of bat echolocation calls in acoustic monitoring: A cautionary note for a sound analysis. Ecological Indicators, 66, 598-602.

https:/ / doi.org/10.1016/j.ecolind.2016.02.036

Rydell, J, Arita, H. T., Santos, M., \& Granados, J. (2002). Acoustic identification of insectivorous bats (order Chiroptera) of Yucatan, Mexico. Journal of Zoology, 257(1), 27-36.

https://doi.org/10.1017/S0952836902000626

Sánchez, L., C.R. Moreno \& E. C. Mora. (2017). Echolocation calls of Natalus primus (Chiroptera: Natalidae): Implications for conservation monitoring of this species. Cogent Biology, 3(1), 1355027.

https:/ / doi.org/10.1080/23312025.2017.1355027

Schmeller, D. S., Weatherdon, L. V., Loyau, A., Bondeau, A., Brotons, L., Brummitt, N., Regan, E. C. (2018). A suite of essential biodiversity variables for detecting critical biodiversity change. Biological Reviews, 93(1), 55-71.

https:/ / doi.org/10.1111/brv.12332

Schnitzler, H.-U., Kalko, E. K. V., Kaipf, I., \& Grinnell, A. D. (1994). Fishing and echolocation behavior of the greater bulldog bat, Noctilio leporinus, in the field. Behavioral Ecology and Sociobiology, 35(5), 327-345.

https:/ / doi.org/10.1007/BF00184422

Schnitzler, H.-U., and E. K. V. Kalko. (2001). Echolocation by insect-eating bats. BioScience, 51(7), 557-569.

https:/ / doi.org/10.1641/0006-3568(2001)051[0557:EBIEB]2.0.CO;2

Shannon, C.E. (1949). Communication in the presence of noise. Proc IRE, 37(1),10-21.
Siemers, B. M. (2004). Bats in the field and in a flight cage: recording and analysis of their echolocation calls and behavior. Bat Echolocation Research: Tools, Techniques, and Analysis, 107-113 Austin, Texas, US.: Bat Conservation International.

Siemers, B. M. (2014). Female mate choice can drive the evolution of high frequency echolocation in bats: a case study with Rhinolophus mehelyi. PLoS One, 9(7), e103452.

https://doi.org/10.1371/journal.pone.0103452

Siemers, B. M., Kalko, E. K. V., \& Schnitzler, H.-U. (2001). Echolocation behavior and signal plasticity in the Neotropical bat Myotis nigricans (Schinz, 1821) (Vespertilionidae): a convergent case with European species of Pipistrellus? Behavioral Ecology and Sociobiology, 50(4), 317-328.

https:/ / doi.org/10.1007/s002650100379

Siemers, B. M., Beedholm, K., Dietz, C., Dietz, I., \& Ivanova, T. (2005). Is species identity, sex, age or individual quality conveyed by echolocation call frequency in European horseshoe bats? Acta Chiropterologica, 7(2), 259-274.

https:/ / doi.org/10.3161/150811005775162579

Sueur, J., Aubin, T., \& Simonis, C. (2008). Seewave: a free modular tool for sound analysis and synthesis. Bioacoustics, 18, 213-226. http:/ / www.tandfonline. com/doi/abs/10.1080/09524622.2008.9753600.

Simmons, N. B. (2005). An Eocene big bang for bats. Science, 307(5709), 527-528.

https:/ / doi.org/10.1126/science.1108871

Stathopoulos, V., Zamora-Gutiérrez, V., Jones, K. E., \& Girolami, M. (2018). Bat echolocation call identification for biodiversity monitoring: a probabilistic approach. Journal of the Royal Statistical Society: Series C (Applied Statistics), 67(1), 165-183.

https://doi.org/10.1111/rssc.12217

Staton, T., \& Poulton, S. (2012). Seasonal variation in bat activity in relation to detector height: A case study. Acta Chiropterologica, 14(2), 401-408.

https://doi.org/10.3161/150811012X661710

Szewczak, J. M. (2004). Advanced analysis techniques for identifying bat species. En Brigham M.R., E.K.V. Kalko, G. Jones, S. Parsons, H. J.G.A. Limpens (Eds.). Bat Echolocation Research: Tools, Techniques and Analysis. Pp 121-126. Austin, Texas: Bat Conservation International.

Tschapka, M., Brooke, A.P., \& Wasserthal, L.T. (2000). Thyroptera discifera (Chiroptera: Thyropteridae): A new record for Costa Rica and observations on echolocation. International Journal of Mammalian Biology. 65(4), 193-198. 
Wilson, D.E., \& Mittermeier, R.A. (Ed.). 2019. Handbook of the mammals of the world: bats. Lynx Edicions, Conservation International, IUCN. Barcelona. $1008 \mathrm{p}$.

Xu, Z., Jing, W., Keping, S., Tinglei, J., Yunlei, J., \& Jiang, F. (2008). Echolocation calls of Rhinolophus ferrumequinum in relation to habitat type and environmental factors. Acta Ecologica Sinica, 28(11), 5248-5258.

https:/ / doi.org/10.1016/S1872-2032(09)60007-X

Yoh, N., Syme, P., Rocha, R., Meyer, C. F., \& López-Baucells, A. (2020). Echolocation of Central Amazonian 'whispering' phyllostomid bats: call design and interspecific variation. Mammal Research, 1-15.

https:/ / doi.org/10.1007/s13364-020-00503-0

Zamora-Gutiérrez, V., López-González, C., MacSwiney González, M. C., Fenton, B., Jones, G., Kalko,
E. K. V., Jones, K. E. (2016). Acoustic identification of Mexican bats based on taxonomic and ecological constraints on call design. Methods in Ecology and Evolution, 7(9), 1082-1091.

https:/ / doi.org/10.1111/2041-210X.12556

Zamora-Gutiérrez, V., Ortega, J., Avila-Flores, R., Aguilar-Rodríguez, P. A., Alarcón-Montano, M., AvilaTorresagatón, L. G., ... \& Chávez-Cauich, M. (2020). The Sonozotz project: Assembling an echolocation call library for bats in a megadiverse country. Ecology and Evolution, 1-16.

https:/ / doi.org/10.1002/ece3.6245

Zurc, D., Guillén-Servent, A., \& Solari, S. (2017). Chillidos de ecolocación de murciélagos Emballonuridae en una sabana xerófila-semiseca del Caribe Colombiano. Mastozoología Neotropical, 24(1), 201-218. 
Anexo 1. Glosario

\begin{tabular}{cl}
\hline Término & \multicolumn{1}{c}{ Definición } \\
\hline $\begin{array}{c}\text { Alternación de } \\
\text { pulsos* }\end{array}$ & $\begin{array}{c}\text { Algunas familias de murciélagos presentan de dos a tres tipos de pulsos. Estos pueden variar en } \\
\text { frecuencia, duración y estructura y generalmente se identifican como alternación de frecuencia } \\
\text { baja, media y alta. Sin embargo, esto varía entre géneros y familias. }\end{array}$ \\
\hline Amplitud & $\begin{array}{l}\text { Volumen de una señal, a menudo medido en decibeles (dB). Para estudios de bioacústica, la ampli- } \\
\text { tud se entiende como la cantidad de energía que el organismo imprime al generar la señal acústica. }\end{array}$ \\
\hline Ancho de banda* & $\begin{array}{l}\text { El ancho de banda es la diferencia entre la frecuencia inicial y la final de un pulso. La banda ancha } \\
\text { se refiere a cambios amplios en frecuencia sobre una duración corta. La banda estrecha significa } \\
\text { que hay poco cambio en frecuencia sobre el tiempo (Fenton, 2010). }\end{array}$ \\
\hline
\end{tabular}

Son las frecuencias múltiplos enteros de una frecuencia fundamental en la señal acústica. Algunas especies emiten pulsos de ecolocalización que presentan mayoritariamente un solo armónico, el Armónico* cual se denomina armónico fundamental, pero otras presentan además estos conjuntos de pulsos sobre y bajo el armónico fundamental, que suele ser el segundo y contener una cantidad de energía mayor si se le compara con los demás armónicos. El estudio de armónicos es importante para la caracterización de ciertas familias (Lisón, 2011).

\begin{tabular}{cl}
$\begin{array}{c}\text { Biblioteca de } \\
\text { referencia }\end{array}$ & $\begin{array}{l}\text { Banco o colección de sonidos de referencia los cuales fueron obtenidos mediante técnicas de gra- } \\
\text { bación que permiten tener certeza que el sonido captado pertenece al individuo en estudio. Estas } \\
\text { bibliotecas no sólo permiten captar la variación inter e intraespecífica de las especies registradas, } \\
\text { sino que facilitan la identificación de especies, mediante la comparación con otras grabaciones. }\end{array}$ \\
\hline
\end{tabular}

Es un método de grabación de señales de ecolocalización de murciélagos, basado en retener la frecuencia con mayor energía en cada segmento de tiempo de progresión de la señal acústica. Se Cruces por cero (Zero utiliza cuando es necesario optimizar el espacio de almacenamiento de datos. Las grabaciones Crossing o ZC) ZC no contienen información de amplitud, ni representan frecuencias de menor amplitud en el mismo segmento temporal de la frecuencia registrada. Esto implica que se pierdan las características del ancho de banda de la señal, como los armónicos.

\begin{tabular}{cl}
\hline $\begin{array}{c}\text { Dirección de } \\
\text { modulación }\end{array}$ & $\begin{array}{l}\text { Hace referencia a la forma ascendente (de una frecuencia inicial baja a una frecuencia final alta) } \\
\text { o descendente (de una frecuencia inicial alta a una frecuencia final baja) en el cambio de frecuen- } \\
\text { cias de un pulso de ecolocalización o de un segmento de este a través del tiempo. }\end{array}$ \\
\hline $\begin{array}{c}\text { División de } \\
\text { frecuencia }\end{array}$ & $\begin{array}{l}\text { Es una técnica para grabar o reproducir en tiempo real una señal ultrasónica, cuya frecuencia, } \\
\text { al ser captada, se divide en múltiplos fijos (10X, 20X, etc), para que puedan ser escuchados por } \\
\text { humanos y/o ser almacenados fácilmente. }\end{array}$ \\
\hline $\begin{array}{c}\text { Duración del pulso } \\
\text { (ms) }\end{array}$ & $\begin{array}{l}\text { Tiempo transcurrido entre el inicio del pulso y el final del pulso. La unidad es el milisegundo } \\
\text { (ms). Para los análisis de duración se utiliza el oscilograma. }\end{array}$ \\
\hline Ecolocalización & Uso de ultrasonido y los ecos de retorno, para orientar y navegar en el ambiente. Se ha \\
& $\begin{array}{l}\text { usado como sinónimo de "ecolocación", pero esta opción no es correcta dado que } \\
\text { "locar" no es un verbo reconocido en castellano, mientras que "localizar" sí. }\end{array}$ \\
\hline Espacio estructural- \\
mente complejo & $\begin{array}{l}\text { En el ámbito acústico, el"clutter" se define como todo aquello que está dentro de un espacio de- } \\
\text { terminado en forma desordenada, que al ser ensonificado genera distintos frentes de eco que } \\
\text { se superponen, y que corresponden a muchas superficies reflejantes. Cuando se dice que es un } \\
\text { espacio estructuralmente complejo, o con un alto clutter, significa que hay muchos objetos en el } \\
\text { espacio de forma desordenada, que para un murciélago implicaría ruido de fondo. Ejemplo, un } \\
\text { bosque con muchos árboles. }\end{array}$ \\
\hline Espacios abiertos & $\begin{array}{l}\text { Contrario al espacio estructuralmente complejo. Representa un espacio con poco clutter, una zona } \\
\text { abierta como un pastizal o por encima del dosel del bosque, a gran altura. }\end{array}$ \\
\hline
\end{tabular}


Término

Espectro completo

(full spectrum)

Espectro de poder

Espectrograma

Estructura del pulso*

Fase de aproximación

Fase terminal (Feeding Buzz)

Forma del pulso

Frecuencia constan-
te $(\mathrm{FC})$

Frecuencia de máxi-
ma energía*

Frecuencia del punto de inflexión

Frecuencia final ${ }^{*}$

Frecuencia inicial ${ }^{*}$

Frecuencia máxima*

\section{Definición}

Es un método de muestreo que captura toda la información de la onda de sonido emitida por el murciélago. El espectro completo describe una grabación de audio que incluye tiempo, frecuencia y amplitud.

Es una representación gráfica de la amplitud de las distintas frecuencias que componen un pulso. Suele usarse para identificar sobre qué frecuencia o grupos de frecuencias se concentra mayor energía en una señal emitida.

Representación visual de una señal acústica. El eje horizontal representa el tiempo, el cual transcurre de izquierda a derecha. En el eje vertical se ubican las frecuencias. La amplitud está representada por la intensidad del color con la que se representa cada pulso de ecolocalización.

Combinación de diferentes segmentos con distinta modulación en una sola señal. Al describir un pulso de un murciélago de la familia Emballonuridae, por ejemplo, su estructura se compone de un segmento FM ascendente, un segmento de FQC y un segmento FM descendente.

Es emitida una vez el murciélago detecta una presa potencial. Usualmente aumenta la tasa de emisión de pulsos y el ancho de banda, y se reduce la duración de los pulsos. Esto le permite al murciélago recopilar información relevante sobre la situación espacial de la presa y ajustar su comportamiento de vuelo para realizar la captura.

Conjunto de pulsos emitidos por el murciélago cuando se desplaza o busca comida. Se caracteriza por presentar parámetros con valores estereotípicos, lo que significa que variables como las frecuencias iniciales y finales, la duración del pulso, del intervalo interpulso, entre otras, se mantienen poco variables. Por esta razón se utilizan los pulsos emitidos en fase de búsqueda para describir e identificar distintas especies.

Esta es emitida una vez concluye la fase de aproximación. El murciélago tiene poco control vocal en su emisión por lo que se trata de una emisión automática, caracterizada por una alta tasa de repetición de pulsos de corta duración, cortos intervalos entre pulsos y modulación muy rápida. Brinda información precisa al murciélago sobre la localización exacta de la presa en el instante previo a su captura. Usualmente se usa el registro de fases terminales o feeding buzzes para estimar la intensidad de forrajeo en estudios de uso de hábitat.

La descripción de un pulso de ecolocalización puede apoyarse en el uso de formas geométricas: pulsos rectos, curvilineares, cóncavos, etc.

Generalmente se refiere a un tipo de pulso cuya frecuencia se mantiene invariable sobre un tiempo relativamente prolongado (>10 ms). La poca variabilidad en las frecuencias componentes de este tipo de pulsos hace que sean de banda estrecha.

También conocida como frecuencia de energía máxima (FME) o frecuencia principal. Es aquella sobre la cual el murciélago concentra la mayor cantidad de energía durante la emisión de la señal de ecolocalización. Este resulta ser un parámetro importante para la identificación de murciélagos, ya que llega a tener valores típicos para una determinada especie o grupo de especies. La desviación estándar de la frecuencia pico permite la detección de especies de llamada alternantes.

Frecuencia o grupo de frecuencias que se encuentran en el punto donde la velocidad de modulación del pulso cambia abruptamente.

Frecuencia con la que termina cada señal de ecolocalización. Puede ser menor a la inicial en pulsos descendentes, mayor a la inicial en pulsos ascendentes.

Frecuencia con la que comienza cada señal de ecolocalización .Puede ser menor a la inicial en pulsos descendentes, mayor a la inicial en pulsos ascendentes.

Frecuencia más alta contenida en la señal de ecolocalización. Equivalente a la frecuencia inicial en pulsos descendentes o a la frecuencia final en pulsos ascendentes. Se mide en kilohertz $(\mathrm{kHz})$. 
Término

Frecuencia mínima* (FM)

\begin{tabular}{c}
\hline Heterodino \\
\hline $\begin{array}{c}\text { Intervalo entre } \\
\text { pulso* }\end{array}$ \\
\hline $\begin{array}{c}\text { Modulación del } \\
\text { pulso }\end{array}$ \\
\hline Número de pulsos*
\end{tabular}

\begin{tabular}{c}
$\begin{array}{c}\text { Número de } \\
\text { secuencias }\end{array}$ \\
\hline Oscilograma
\end{tabular}

Parámetros acústicos

Frecuencia más baja contenida en la señal de ecolocalización. Equivalente a la frecuencia final en pulsos descendentes o a la frecuencia inicial en pulsos ascendentes. Se mide en kilohertz $(\mathrm{kHz})$.

Generalmente se refiere a un tipo de pulsos que contienen un amplio conjunto de frecuencias banda ancha, las cuales cambian en un segmento de tiempo muy corto (1-5 ms). Típicamente van de frecuencias iniciales relativamente altas a frecuencias finales relativamente bajas en pulsos FM descendentes, y viceversa para pulsos FM ascendentes.

Técnica que combina una frecuencia de una señal de ecolocalización de entrada, con una frecuencia fija generada por un oscilador interno en el dispositivo de detección, resultando en un sonido audible para humanos. Generalmente los dispositivos de detección que usan esta técnica son de banda estrecha.

Es el tiempo que transcurre entre el inicio de un pulso de ecolocalización y el inicio del pulso subsecuente. Medido en milisegundos (ms).

Es el cambio de frecuencias componentes de un pulso de ecolocalización frente al tiempo de progresión de este. Cuando el cambio de frecuencias se hace sobre una banda ancha en lapsos de poca duración, se dice que es un pulso muy modulado o de modulación rápida (FM). Por otro lado, cuando el cambio de frecuencias de un pulso se hace sobre una banda estrecha en lapsos de alta duración, se dice que es un pulso poco modulado, de modulación lenta o de frecuencia cuasiconstante (FQC).

Cantidad de pulsos que contiene una secuencia de ecolocalización. En bioacústica, este término se refiere a los pulsos que componen la fase de búsqueda analizados para una especie en particular.

En los estudios de diversidad de murciélagos donde se emplea la bioacústica, siempre es necesario registrar el número de secuencias que se analizan, como si estas fueran un " $\mathrm{n}$ " o un número de muestras.

Representación gráfica de una forma de onda de audio. El tiempo se muestra en el eje horizontal, y la amplitud se muestra en el eje vertical.

Son todas las variables relevantes que se miden de las señales de ecolocalización de los murciélagos. Estos pueden ser del dominio espectral como la frecuencia o la amplitud, o del dominio temporal como la duración o el intervalo interpulso.

Es la amplitud en $\mathrm{dB}$ que tiene la frecuencia con mayor energía dentro del pulso. Usualmente es útil para discriminar especies que usan la misma banda de frecuencias.

\section{Señal acústica Una emisión discreta de sonido, equivalente a un pulso.}

Tasa de muestreo

Determina la cantidad de bits que debe muestrear un dispositivo por unidad de tiempo. Esta debe ser el doble de la frecuencia máxima que se desea muestrear (Teorema de Nyquist-Shannon), pues solo así se puede lograr una muestra representativa de una señal acústica con muchas oscilaciones por unidad de tiempo (alta frecuencia).

Ventana

Es uno de los atributos que se configuran para la representación gráfica de señales acústicas, con el fin de evitar la discontinuidad entre los bloques de información analizados. Se utiliza en hamming los programas de análisis acústico para "suavizar" la imagen de los pulsos. Usualmente se toma como el estándar para la representación de señales acústicas de murciélagos. 


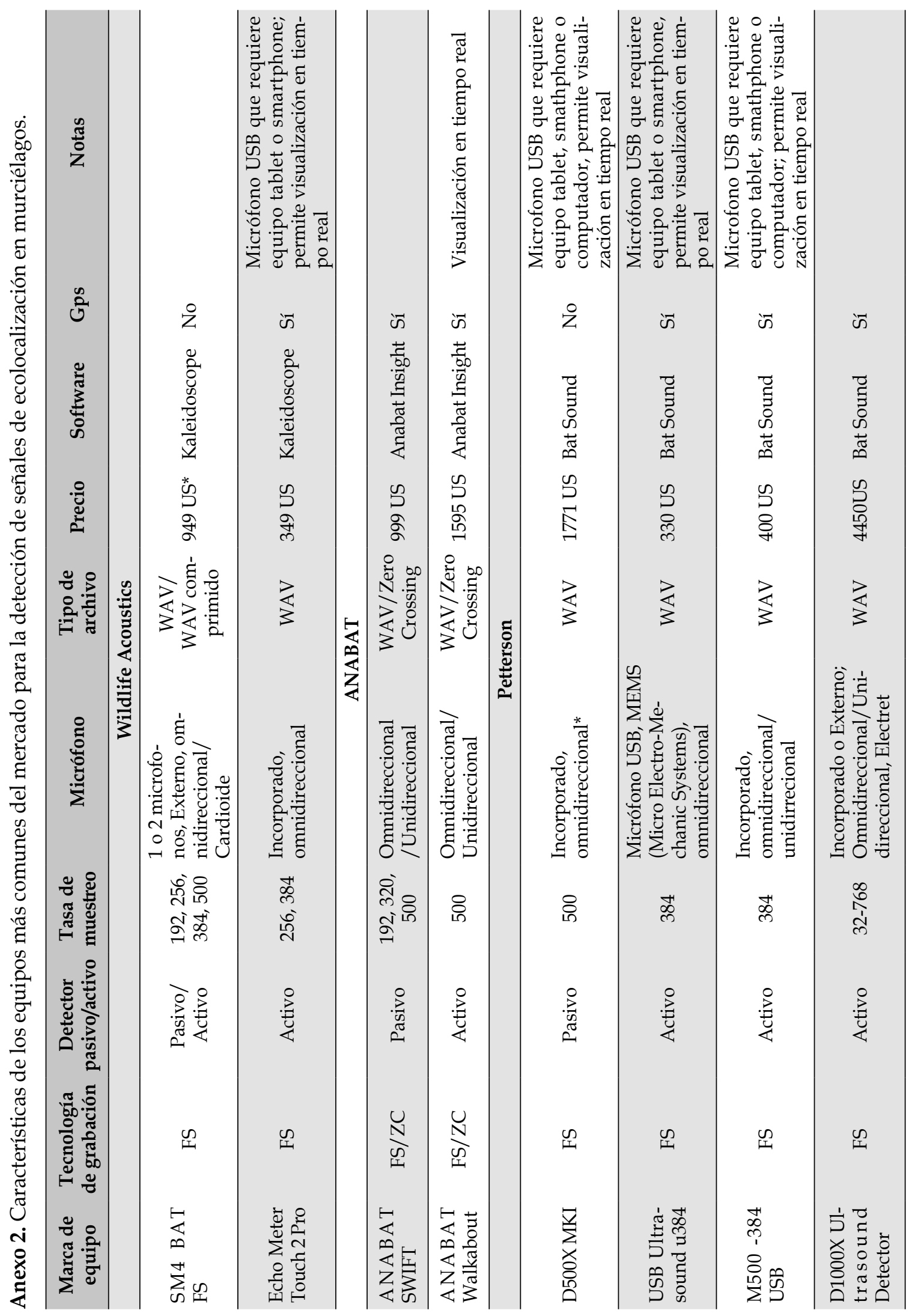




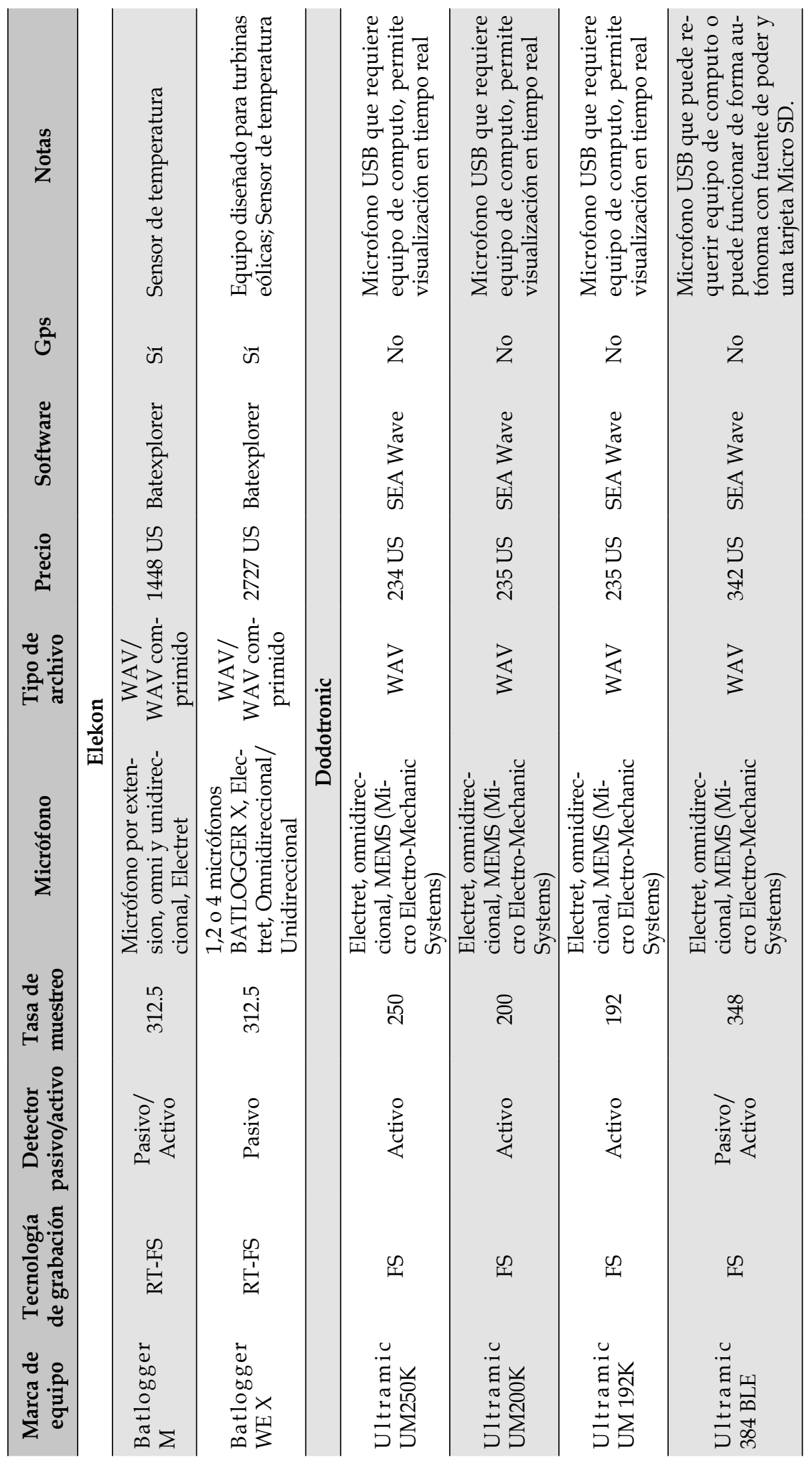




\section{Daniela Martínez-Medina}

Fundación Reserva Natural La Palmita, Centro de Investigación

Bogotá, Colombia

danimmo87@gmail.com

https:/ / orcid.org/0000-0002-9431-8399

Jefferson Sánchez

Cuántico Global Eco Services

Medellín, Colombia

jefferson.sanchez1@correo.uis.edu.co

https:/ / orcid.org/0000-0002-8652-1776

\section{Danny Zurc}

Centro de Ciencia Museo de Ciencias Naturales de La Salle Instituto Tecnológico Metropolitano

Medellín, Colombia

curaduriamuseo@itm.edu.co

https:/ / orcid.org/0000-0003-4830-281X

\section{Francisco Sánchez}

Universidad de los Llanos

Villavicencio, Colombia

fsanchezbarrera@unillanos.edu.co

https:/ / orcid.org/0000-0001-8826-5692

\section{Aída Otálora-Ardila}

Instituto de Investigación de Recursos Biológicos

Alexander von Humboldt

Villa de Leyva, Colombia

aotalora@gmail.com

https://orcid.org/0000-0002-0020-7509

\section{Carlos Restrepo-Giraldo}

Sonoran Institute

Mexicali, México

carlos.restrepo.giraldo@gmail.com

https:/ / orcid.org/0000-0003-4111-2669

\section{Orlando Acevedo-Charry}

Instituto de Investigación de Recursos Biológicos Alexander von Humboldt

Villa de Leyva, Colombia

oacevedo@humboldt.org.co

https:/ / orcid.org/0000-0003-4964-8994

\section{Fabián Hernández Leal}

Universidad de los Llanos

Villavicencio, Colombia

orlando.hernandez@unillanos.edu.co

https://orcid.org/0000-0003-1545-0349

\section{Diego Lizcano}

The Nature Conservancy

Bogotá, Colombia

diego.lizcano@tnc.org

https:/ / orcid.org/0000-0002-9648-0576

Estándares para registrar señales de ecolocalización y construir bibliotecas de referencia de murciélagos en Colombia

Citación del artículo: Martínez-Medina, D. Sánchez, J., Zurc, D., Sánchez, F., Otálora-Ardila, A., Restrepo-Giraldo, C., Acevedo-Charry, O., Hernández-Leal, F. \& Lizcano, D.(2021). Estándares para registrar señales de ecolocalización y construir bibliotecas de referencia de murciélagos en Colombia. Biota Colombiana, 22(1), 36-56

https:/ / doi.org/10.21068/ c2021.v22n01a03

Recibido: 31 de mayo 2020

Aceptado: 15 de noviembre 2020 